\title{
FACTORES DE RIESGO ASOCIADOS A LA MORBIMORTALIDAD EN ADOLESCENTES EMBARAZADAS QUE ACUDEN AL CENTRO DE SALUD METROPOLITANO - TACNA EN LOS AÑOS 2002 AL 2004
}

Responsable: Obst. Juana Barreda Grados Miembro: Lic. Obst. Bernardino Damián López

\section{RESUMEN}

El propósito de la presente investigación se centra en identificar los factores de riesgo asociados a la morbimortalidad durante el embarazo en adolescentes que acuden al C.S. Metropolitano durante el periodo comprendido entre el $2002 \mathrm{y}$ el 2004, en el departamento de Tacna.

El diseño de investigación es no experimental, transversal descriptivo. La muestra se ha calculado con un $95 \%$ de confianza y un $5 \%$ de error; el tipo de muestreo ha sido no probabilistica consecutivo.Se ha utilizado como instrumento de recolección de datos un cuestionario semiestructurado de 17 items.

Se ha concluído que los factores de riesgo presentes en las adolescentes embarazadas que en mayor proporción se presentan son las dimensiones sociales y las infecciones vaginales.

\section{ABSTRACT}

The research is centered on the identification of de risk factors wich are associated to the morbility and mortality during pregnancy in adolescents, who got to the C.S. Metropolitano during the period 2002 to 2004 in the department of Tacna. The design of the investigation is no experimental, transversal descriptive. The sample has been calculated with a $95 \%$ of confidence and $5 \%$ of error, the type of sampling has been not probabilistic consecuted. The semi-structured questionnaire wich has 17 items, has been used as an instrument to collect data.

As a conclusion, the risk factors are presented in pregnancy adolescents, that factors are presented in bigger proportion , are the social dimension and the urinary infections.

\section{INTRODUCCIÓN}

La población adolescente de Tacna es de 81,894 que equivale al $28,72 \%$ de la población total. Los problema de salud que afrontan los adolescentes no siempre se reflejan en cifras, como el caso de incremento de consumo de sustancias psicoactivas y otros, porque no hay registros en ninguna institución, sin embargo los embarazos no deseados, partos, el aborto y sus complicaciones terminan en instituciones de salud. El parto en adolescentes se ha incrementado en el último quinquenio, de $12,5 \%$ a $16 \%$.

Según ENDES 2000, el $11 \%$ de las adolescentes de 15 a 19 años ya eran madres, y más de un tercio de ellas no cuenta con ningún grado de educación, poniendo en riesgo su vida y la de sus hijos, ya que está demostrado que la salud, educación y nutrición de las madres es un factor decisivo para la supervivencia y desarrollo pleno de la niñez.

Del total de gestantes en los servicios de salud del Ministerio de Salud, 15\% fueron adolescentes. En el análisis de estos datos se deben tomar en consideración las coberturas realizadas por el seguro escolar gratuito que, en 1998, realizó 3,015 controles prenatales y atendió 1440 partos de adolescentes. El embarazo en adolescente es una situación condicionante de alto riesgo obstétrico y perinatal, ya que se asocia a diversas factores sociales y económicos como a patologías del embarazo, parto y del recién nacido. En consecuencia, los problemas orgánicos derivados del embarazo en la adolescencia pueden ser solucionados con un sistema de atención médico multidisciplinario.

En la región de Tacna, la mortalidad perinatal es de 10 por 1000 embarazos durante el periodo de 1995 al 2000; esta cifra es inferior comparada al promedio nacional que es de 23 por 1000 embarazos por año.

El embarazo en adolescentes en la región de Tacna fue de $7 \%$ el año 2000 con relación al total de embarazos registrados; esta cifra también resulta inferior con relación al promedio nacional que es de $10 \%$ según la Oficina General de Epidemiología del Ministerio de Salud.

En contraste, observamos que en EEUU los índices de embarazos en adolescentes registrados ha declinado desde el 1991, constituyendo en el 2002 alrededor del $11 \%$ de todos los nacimientos en nuestro país. Auchtre y col. Reportan que las patologías asociadas al embarazo, como hipertensión arterial, amenaza de parto prematuro, desprendimiento prematuro de placente, rotura prematura de membranas y retardo de crecimiento intrauterino, se observaron en el $50 \%$ de los casos y en el $33 \%$ de los controles. En el caso de Perú, Alarcón señala que existen indicios de que la mayoria de las madres adolescentes dejan los estudios antes de embarazarse; asimismo sólo el $63 \%$ culminó los estudios primarios, mientras que entre las madres adultas el $81 \%$ también logró este nivel educativo.

La situación de las madres adolescentes en el Perú es distinta a la de otros países. El embarazo adolescente es producto de las ventajas en las que vive y se desarrolló esta madre.

\section{MATERIALY MÉTODOS}

La población objeto de estudio la componen todas las gestantes adolescentes que cumplieron con los 
criterios de inclusión y que accedieron al control prenatal durante los años 2002-2004 en el Centro de Salud Metropolitano del MINSA. El tamaño de muestra se definió considerando un nivel de confianza del $95 \%(Z=1,96)$ y un error del $5 \%$, calculando en 90 .

El instrumento utilizado fue un cuestionario semiestructurado de 17 item con preguntas abiertas y dicotómicas, que comprendía las variables: características de las adolescentes, factores de riesgo preconcepcionales y factores de riesgo que aparecen durante el curso del embarazo, AUDIT. (Test de Identificación de transtornos relacionados con el uso de alcohol).

\section{RESULTADOS:}

Variable: Características de la gestante adolescente.

CUADRO N ${ }^{\circ} 1$ Edad de las Gestantes Adolescentes

\begin{tabular}{|c|c|c|c|c|c|c|c|c|}
\hline \multirow{2}{*}{ EDAD } & \multicolumn{2}{|c|}{2002} & \multicolumn{2}{|c|}{2003} & \multicolumn{2}{c|}{$\mathbf{2 0 0 4}$} & \multicolumn{2}{c|}{ TOTAL } \\
\cline { 2 - 9 } & $\mathbf{N}^{\mathbf{0}}$ & $\%$ & $\mathbf{N}^{\circ}$ & $\%$ & $\mathbf{N}^{\circ}$ & $\%$ & $\mathbf{N}^{\circ}$ & $\%$ \\
\hline Sin dato & 1 & 3,3 & & 0,0 & 0 & 0,0 & 1 & 0,8 \\
\hline 15 & 1 & 3,3 & 2 & 4,7 & 2 & 3,7 & 5 & 3,9 \\
\hline 16 & 2 & 6,7 & 7 & 16,3 & 9 & 16,7 & 18 & 14,2 \\
\hline 17 & 7 & 23,3 & 7 & 16,3 & 11 & 20,4 & 25 & 19,7 \\
\hline 18 & 7 & 23,3 & 6 & 14,0 & 8 & 14,8 & 21 & 16,5 \\
\hline 19 & 12 & 40,0 & 21 & 48,8 & 24 & 44,4 & 57 & 44,9 \\
\hline TOTAL & 30 & 100,0 & 43 & 100,0 & 54 & 100,0 & 127 & 100,0 \\
\hline
\end{tabular}

Fuente: Historia Clinica C.S. Metropolitano - Tacna 2004

\section{Interpretación:}

En el Cuadro 1 se observa que el $44,9 \%$ de las adolescentes tiene la edad de 19 , el $19,7 \%$ tiene una edad de 17 años, el $16.5 \%$ tiene una edad de 18 años y el $14,2 \%$ tiene una edad promedio de 16 años.

Factores de riesgo preconcepcionales: Antecedentes Socioeconómicos.

CUADRO N ${ }^{\circ} 2$ Estado Civil de las Gestantes Adolescentes

\begin{tabular}{|l|r|r|r|r|r|r|r|r|}
\hline \multirow{2}{*}{$\begin{array}{c}\text { ESTADO } \\
\text { CIVIL }\end{array}$} & \multicolumn{2}{|c|}{2002} & \multicolumn{2}{|c|}{2003} & \multicolumn{2}{|c|}{2004} & \multicolumn{2}{|c|}{ TOTAL } \\
\cline { 2 - 9 } & $\mathbf{N}^{\circ}$ & $\%$ & $\mathbf{N}^{\circ}$ & \multicolumn{1}{c|}{$\%$} & $\mathbf{N}^{\circ}$ & \multicolumn{1}{c|}{$\%$} & $\mathbf{N}^{\circ}$ & \multicolumn{1}{c|}{$\%$} \\
\hline Soltera & 17 & 56.7 & 22 & 51.2 & 28 & 51.9 & 67 & 52.8 \\
\hline Conviviente & 9 & 30.0 & 20 & 46.5 & 23 & 42.6 & 52 & 40.9 \\
\hline Casada & 2 & 6.7 & 0 & 0.0 & 2 & 3.7 & 4 & 3.1 \\
\hline sin dato & 2 & 6.7 & 1 & 2.3 & 1 & 1.9 & 4 & 3.1 \\
\hline TOTAL & 30 & 100.0 & 43 & 100.0 & 54 & 100.0 & 127 & 100.0 \\
\hline
\end{tabular}

Fuente: Historia Clinica C.S. Metropolitano - Tacna 2004

\section{Interpretación:}

En el Cuadro 2 se observa que el $52,8 \%$ de las gestantes adolescentes son solteras; el $40,9 \%$ son convivientes; el $3,1 \%$ son casadas, lo que constituye un factor de riesgo.

Factores de riesgo concepcionales: Patologia maternas en gestantes adolescentes

CUADRO N ${ }^{\circ} 3$ Patologias Maternas en Gestantes Adolescentes

\begin{tabular}{|c|c|c|c|c|c|c|c|c|}
\hline \multirow[b]{2}{*}{ EXAMEN } & \multicolumn{2}{|c|}{2002} & \multicolumn{2}{|c|}{2003} & \multicolumn{2}{|c|}{2004} & \\
\hline & $\mathrm{N}^{\circ}$ & $\%$ & $\mathrm{~N}^{2}$ & $\%$ & $\mathrm{~N}^{\circ}$ & $\%$ & $\mathrm{~N}^{\circ}$ & $\%$ \\
\hline Anemia & 3 & 7.5 & 8 & 14,3 & 12 & 12.5 & 23 & 12,0 \\
\hline Infección urinaria & 14 & 35,0 & 16 & 28,6 & 30 & 31,3 & 60 & 31,3 \\
\hline Infección vaginal & 8 & 20,0 & 16 & 28,6 & 25 & 26,0 & 49 & 25,5 \\
\hline Amenaza de aborto & 1 & 2,5 & 1 & 1,8 & 3 & 3,1 & 5 & 2,6 \\
\hline Emesis gravidica & 3 & 7,5 & 0 & 0,0 & 3 & 3,1 & 6 & 3,1 \\
\hline Amenaza de parto prematuro & 1 & 2,5 & & 0,0 & & 0,0 & 1 & 0,5 \\
\hline Aborto & 0 & 0,0 & 2 & 3,6 & 1 & 1,0 & 3 & 1,6 \\
\hline Podalica a 38 sem & 2 & 5,0 & 2 & 3,6 & 3 & 3,1 & 7 & 3,6 \\
\hline Aumento exagerado de peso matemo & 3 & 7,5 & 3 & 5,4 & 5 & 5,2 & 11 & 5,7 \\
\hline Poca ganancia peso matemo & 1 & 2,5 & 4 & 7,1 & 5 & 5,2 & 10 & 5,2 \\
\hline $\begin{array}{l}\text { Otras ( gastritis, depresión, faringitis, } \\
\text { demmatits, polihidramnios leve, elc.) }\end{array}$ & 4 & 10,0 & 4 & 7,1 & 9 & 9,4 & 17 & 8,9 \\
\hline & 40 & 100,0 & 56 & 100,0 & 96 & 100,0 & 192 & 100,0 \\
\hline
\end{tabular}

Fuente: Historia Clínica C.S. Metropolitano Tacna - 2004
Interpretación.-

En el Cuadro 3 se observa que el $31,3 \%$ de las gestantes adolescentes han cursado con inyección urinaria; el 25,5 tenido inyección vaginal y el $12,0 \%$ tuvieron anemia. El $5,7 \%$ tuvieron aumento exagerado de peso materno y el 5,2 tuvieron poca ganancia de peso materno. Los resultados evidencian que mayoritariamente las infecciones urinarias, en los tres años de estudios, alcanzan la proporción más alta en relación a las demás patologias.

Factores de riesgo concepcionales: Promedio de ganancia de peso materno

CUADRO N ${ }^{\circ} 4$ Promedio de Ganancia de Peso Materno en Gestantes Adolescentes

\begin{tabular}{|l|r|r|r|r|r|r|r|r|}
\hline \multirow{2}{*}{ PESO (KILOS) } & \multicolumn{2}{|c|}{$\mathbf{2 0 0 2}$} & \multicolumn{2}{|c|}{$\mathbf{2 0 0 3}$} & \multicolumn{2}{|c|}{$\mathbf{2 0 0 4}$} & \multicolumn{2}{|c|}{ TOTAL } \\
\cline { 2 - 9 } & $\mathbf{N}^{\circ}$ & $\%$ & $\mathbf{N}^{\circ}$ & \multicolumn{1}{c|}{$\%$} & $\mathbf{N}^{\circ}$ & $\%$ & $\mathbf{N}^{\circ}$ & $\%$ \\
\hline Menos de 6 kilos & 1 & 5,0 & 3 & 13,0 & 5 & 10,6 & 9 & 10,0 \\
\hline 6-8 kilos & 1 & 5,0 & 9 & 39,1 & 12 & 25,5 & 22 & 24,4 \\
\hline 9 a 12 kilos & 1 & 60,0 & 8 & 34,8 & 18 & 38,3 & 38 & 42,2 \\
\hline $13-16$ kilos & 4 & 20,0 & 1 & 4,3 & 6 & 12,8 & 11 & 12,2 \\
\hline Más de 17 kilos & 2 & 10,0 & 2 & 8,7 & 6 & 12,8 & 10 & 11,1 \\
\hline TOTAL & 20 & 100,0 & 23 & 100,0 & 47 & 100,0 & 90 & 100,0 \\
\hline
\end{tabular}

Fuente: Historia Clínica C.S. Metropolitano - Tacna 2004

\section{Interpretación:}

En el Cuadro se puede observar que el $42,2 \%$ de las gestantes adolescentes ganó entre 9 - 12 kilos durante el embarazo; el 12,2\% ganó entre 13 - 16 kilos; el $24,4 \%$ ganó entre 6 a 8 kilos y el $11,1 . \%$ Ganó más de 17 kilos.

\section{IV.CONCLUSIONES}

1. En relación con la variable de estudio caracteristicas de las adolescentes, la edad es un factor de riesgo de las gestantes adolescentes, el $60 \%$ entre 15 a 18 años en el periodo de estudio y el $40 \%$ entre 18 y 19 a ñ $0 \mathrm{~s}$

2. Respecto a la variable factores de riesgo preconcepcionales, se evidencia que en los antecedentes socioeconómicos uno de los más elevantes es el estado civil, encontrándose que en los tres años de estudios la proporción de adolescentes embarazadas solteras supera el $50 \%$, lo cual incide en su estabilidad emocional e incrementa la dependencia económica respecto aterceros.

3. En lo que concierne a la variable factores de riesgo que aparecen durante el curso del embarazo, se enfatiza la dimensión patología materna en gestantes adolescentes, concluyéndose que en el 2002, 2003 y 2004 la proporción de infecciones urinarias es de $35 \%$, $28,6 \%$ y $31,3 \%$ respectivamente.

\section{VI.BIBLIOGRAFÍA}

Alarcón , Giovann. El caso de las madres.

Portilla, Arturo, SOTO, Graciela (1995). Educación sexual para adolescentes. CEDER. Edit. Mundo, Arequipa, Perú.

Yotelo, Virgina, LUEGO, Ximena (2002) Adolescencia: Tiempo de decisiones, Manual para trabajo en adolescentes. Editorial Mediterráneo, Chile. 